\title{
Correction to: RECODE: revision control for digital images
}

\section{Fabio Calefato ${ }^{1} \cdot$ Giovanna Castellano $^{1}$ (D) $\cdot$ Veronica Rossano $^{1}$}

Published online: 14 November 2019

(C) Springer Science+Business Media, LLC, part of Springer Nature 2019

\section{Correction to: Multimedia Tools and Applications (2019) https://doi.org/10.1007/s11042-019-7735-9}

The author regrets that the acknowledgment of the original publication contains an error. The correct acknowledgement is written below:

Acknowledgements This work is partially funded by the project "Creative Cultural Collaboration" (C3) under the Apulian INNONETWORK programme, Italy. The authors thank the students Davide Mantellini, Gabriele Neglia, and Salvatore Vestita for developing the code of the RECODE tool as part of their thesis project.

Giovanna Castellano

giovanna.castellano@uniba.it

Fabio Calefato

fabio.calefato@uniba.it

Veronica Rossano

veronica.rossano@uniba.it

1 Computer Science Department, University of Bari, Via Orabona 4, 70125 Bari, Italy 